\title{
Compósitos Magnéticos \\ Poliméricos Nanoestruturados e seu uso como Carreadores de Fármacos Anti- Carcinogênicos
}

\author{
Karyn Nathallye de Oliveira Silva*; (Graduanda em Engenharia Química \\ naUniversidade Federal Rural do Semi-Árido - UFERSA) \\ Francisca Jucileuda da Silva Sousa; (Instituto Federal do Rio Grande do Norte - IFRN) \\ Wogelsanger Oliveira Pereira; (Departamento de Ciências Biomédicas da Faculdade de Ciências da Saúde da \\ UERN) \\ Marco Antonio Morales Torres; (Departamento de Física Teórica e Experimental, Universidade Federal do \\ Rio Grande do Norte) \\ *karyn_nathallye@hotmail.com
}

\section{resumo:}

Devido aos efeitos nocivos de fármacos antineoplásicos sobre tecidos sadios, novos métodos estão sendo desenvolvidos para a vetorização desses fármacos. A vetorização magnética consiste em direcionar nanopartículas de compósitos magnéticos através do fluxo sanguíneo até a região do tumor, usando um gradiente de campo magnético externo. Dessa forma, o objetivo deste trabalho é sintetizar partículas de quitosana funcionalizadas com o polímero Pluronic ${ }^{\circledR}$ F127 e nanopartículas de magnétita para a vetorização magnética do fármaco Doxorrubicina. Após a preparação das partículas foi feito um estudo sobre a cinetica de liberação do fármaco. A preparação das nanopartículas de magnetita foi feita através de dois métodos. O primeiro consiste na oxidação e precipitação de íons de $\mathrm{Fe}^{2+}$ em meio alcalino de $\mathrm{KOH}$ e $\mathrm{KNO}_{3}$, e em atmosfera de gás de nitrogênio, o segundo método consiste na mistura estequiometrica de íons de $\mathrm{Fe}^{2+}$ e $\mathrm{Fe}^{3+}$ em meio alcalino de $\mathrm{NaOH}$. As nanopartículas de magnetita foram encapsuladas na matriz da quitosana usando um método desenvolvido em laboratório. As amostras foram caracterizadas usando as técnicas de difração de raios-X (DRX), espectroscopia Mössbauer (EM) do ${ }^{57} \mathrm{Fe}$, espalhamento dinâmico de luz (EDL), e espectrofotometria de UVVis. As medidas de DRX e EM realizadas em 300K mostraram a formação de nanoparticulas de magnetita com diâmetro de 7 e $30 \mathrm{~nm}$. As medidas de EDL mostraram distribuições de tamanho das partículas de magnetita revestidas com quitosana com diâmetro médio de 250 e 450nm. A doxorrubicina foi encapsulada nas partículas com diâmetro menor. Esse sistema apresenta potencial como terapia alternativa para o tratamento de pacientes com neoplasias.

palavras-chave:

Câncer, Magnetita, Quitosana, Doxorrubicina, Nanopartículas.

Espaço reservado para organização do congresso. 


\section{I ntrodução}

A tecnologia de liberação controlada de fármacos vem se expandindo nos últimos anos. Para o encapsulamento de fármacos geralmente são utilizados poliméros, e materiais inorgânicos como vidros e cerâmicas funcionalizados com moléculas orgânicas.

Na medicina, diversas pesquisas têm sido feitas visando encontrar o melhor método para inserir fármacos em regiões desejadas, e compostos magnéticos nanoestruturados surgem em meio a este contexto proporcionando diversas vantagens. Esses compostos são desejáveis no tratamento de pacientes com câncer por possibilitar o transporte das drogas neoplásicas até o local onde está localizado o tumor, facilitando assim as concentrações necessárias do fármaco além de minimizar os efeitos colaterais em tecidos sadios do corpo. Os compostos nanométricos magnéticos são guiados utilizando um gradiente de campo magnético externo

Um tipo desses compósitos consiste em um núcleo magnético envolvido por um material polimérico. O material polimérico utilizado nesse trabalho é a quitosana, que é um biopolímero, não tóxico e biodegradável [1]. O material magnético utilizado é a magnetita, um óxido de ferro biocompatível e biodegradável [2], que possui um alto valor de magnetização, que facilita sua interação com um campo magnético externo.

Fármacos são levados até as células cancerígenas através dos interstícios entre as células endoteliais que formam as paredes de vasos sanguíneos [3]. Dessa forma, carreadores com tamanhos médios de $200 \mathrm{~nm}$ podem permear as paredes de vasos sanguíneos, abandonando o sistema circulatório para assim ter acesso às células tumorais, propiciando a liberação do fármaco no local desejado.

Este trabalho visa produzir sistemas poliméricos magnéticos nanoestruturados que apresentam poros capazes de encapsular nanopartículas de magnetita e o fármaco doxorrrubicina (Dox). Faremos estudos da cinética da liberação do fármaco em meio aquoso para duas amostras com concentrações diferentes de Dox.

\section{Materiais e métodos}

\subsection{Métodos de caracterização}

Os compósitos foram caracterizados usando as técnicas de Difração de raios-X (DRX), (MiniflexII-Rigaku-Japão); Espectroscopia Mössbauer (Wiessel-Alemanhã), Espectrofotômetria de UvVis (Shimadzu- Japão), Espalhamento Dinâmico de luz (EDL) (Nanotrac- USA).

\subsection{Materiais}

A quitosana utilizada nesse trabalho possui grau de desacetilação de $85 \%$. A solução de quitosana foi preparada à temperatura ambiente dissolvendo-se $8 \mathrm{~g}$ de quitosana em $400 \mathrm{ml}$ de solução aquosa de ácido cítrico $\left(\mathrm{C}_{6} \mathrm{H}_{8} \mathrm{O}_{7} \cdot \mathrm{H}_{2} \mathrm{O}\right)$ à 5 w.\%. A solução permaneceu sob agitação magnética por um período de 12 hs, após esse tempo a solução foi filtrada e mantida sob refrigeração. O pH final da solução ficou em torno de 2,5. Também foram utilizados Pluronic $^{\circledR}$ (Sigma-Aldrich), trietilamina (Sigma-Aldrich), sodium tripolyphosphate (TPP) (Sigma-Aldrich), clorhidrato de doxorrubicina (Dox) (Laboratório Bergamo).

\subsection{Métodos de preparação das amostras 2.3.1 Síntese da magnetita}

A água destilada usada na preparação da magnetita foi degaseificada com gás nitrogênio por duas horas. A magnetita foi sintetizada por dois métodos. Método de síntese 1 [4]: foram preparadas as soluções de 3,1168 g de hidróxido de potássio (KHO) em 20 ml de água; 8,08 g de nitrato de 
potássio $\left(\mathrm{KNO}_{3}\right)$ em $40 \mathrm{ml}$ de água; 2,7792 g de sulfato de ferro $\left(\mathrm{FeSO}_{4}\right)$ em $24 \mathrm{ml}$ de água. Essas soluções foram adicionadas a $315 \mathrm{ml}$ de água. A mistura resultante foi aquecida em banho maria até $90^{\circ} \mathrm{C}$ por 4 horas. Em seguida, a dispersão foi resfriada e as partículas de magnetita foram lavadas na centrífuga para obter ph neutro e por fim foi colocada para secar na estufa a $90^{\circ} \mathrm{C}$.

Método de síntese 2: Foi preparado $100 \mathrm{ml}$ de uma solução de $\mathrm{NaOH} 4 \%$. O pH final foi de 13,01. Logo depois, foi preparada $20 \mathrm{ml}$ de uma solução aquosa que continha 2,411 g de nitrato férrico e 0,801 g de sulfato ferroso. A solução contendo os íons de $\mathrm{Fe}$ foi adicionada à solução de $\mathrm{NaOH}$ e agitada por 15 minutos, as nanopartículas de magnetita mudaram a cor da solução de marron para preto. A mistura final apresentou um valor de $\mathrm{pH}=$ 12,98, em seguida as nanopartículas foram lavadas e separadas na centrífuga até seu pH se tornar neutro. A amostra foi colocada para secar na estufa a $90^{\circ} \mathrm{C}$ por 1 hora.

\subsubsection{Funcionalização da quitosana com a magnetita}

A quantidade de 0,2 mg da magnetita preparadas pelos métodos 1 e 2 foram dispersas em $52 \mathrm{ml}$ de solução de quitosana para funcionalização com o polímero, e permaneceram sob agitação mecânica por 1 hora. Depois, foram levadas a centrífuga para a separação do meio líquido, o sobrenadante foi descartado.

As partículas de magnetita revestidas com a quitosana foram colocadas em uma solução que continha $156 \mathrm{ml}$ de solução de quitosana, 62,4 ml de água destilada e 31,2 mg de TPP. A mistura resultante foi levada ao ultrassom e, depois, foi agitada por 4 horas. Terminado esse período, a dispersão foi centrifugada e o sobrenadante foi descartado. As partículas de magnetita+quitosana resultante foram dispersas em uma solução contendo $100 \mathrm{ml}$ de água destilada e $400 \mathrm{mg}$ de Pluronic ${ }^{\circledR}$ (responsável por aumentar a hidrofilicidade da quitosana em água). A solução final foi levada ao agitador mecânico por aproximadamente 11 horas. Então, as partículas foram recuperadas com a ajuda de um ímã. Os nomes dessas partículas foram de Amostra1 (magnetita preparada pelo método 1) e Amostra2 (magnetita preparada pelo método 2).

\subsection{Encapsulamento da doxorrubicina}

De posse das partículas magnéticas revestidas com quitosana, foram preparadas duas amostras contendo Dox, ver a tabela 1. A doxorrubicina, inicialmente hidrofílica, foi tratada com trietilamina com o objetivo de deprotoná-la, e assim facilitar sua solubilidade em cloroformio. A mistura foi deixada sob agitação por 12 horas, tempo suficiente para evaporação do cloroformio, a seguir foi adicionada a água, então a suspensão foi centrifugada e o sobrenadante foi descartado, reservando o material sólido. Foi acrescentado $2 \mathrm{ml}$ de água milliQ ao material sólido e foi iniciado o estudo da cinética de liberação do fármaco para tempo diferentes. Esse estudo foi feito com ajuda de um espectrofotômetro, antes de cada medida as partículas foram separadas do líquido utilizando a centrífuga. Então, foi medida a absorção do sobrenadante para um feixe de radiação eletromagnetica com comprimento de onda fixo de $480 \mathrm{~nm}$. 


\begin{tabular}{|c|c|c|c|c|c|}
\hline & $\begin{array}{c}\text { Dox } \\
(\mathrm{mg})\end{array}$ & $\begin{array}{c}\text { Partículas } \\
(\mathrm{mg})\end{array}$ & $\begin{array}{c}\text { Trietilamina } \\
(\mu \mathrm{l})\end{array}$ & $\begin{array}{c}\text { Água } \\
(\mathrm{ml})\end{array}$ & $\begin{array}{c}\text { Cloroformio } \\
(\mathrm{ml})\end{array}$ \\
\hline AmostraDox1 & 7,5 & 10,0 & 19,2 & 1.0 & 7,5 \\
\hline AmostraDox2 & 0,9 & 2,5 & 2,5 & 0,7 & 1,0 \\
\hline
\end{tabular}

Tabela 01 - Amostras contendo as partículas de quitosana + magnetita e o fármaco antineoplásico doxorrubicina .

\section{5 - Determinação da oncentração de Dox utilizando dados de medidas espectrofotômetricas}

Para relacionar os valores de absorção com a concentração do fármaco, foram feitas medidas da absorção em várias amostras com concentrações conhecidas. A figura 1 mostra os dados obtidos nas medidas e os resultados do ajuste linear. O coeficiente ângular obtido do ajuste foi de $8,497 \mathrm{ml} / \mathrm{mg}$.

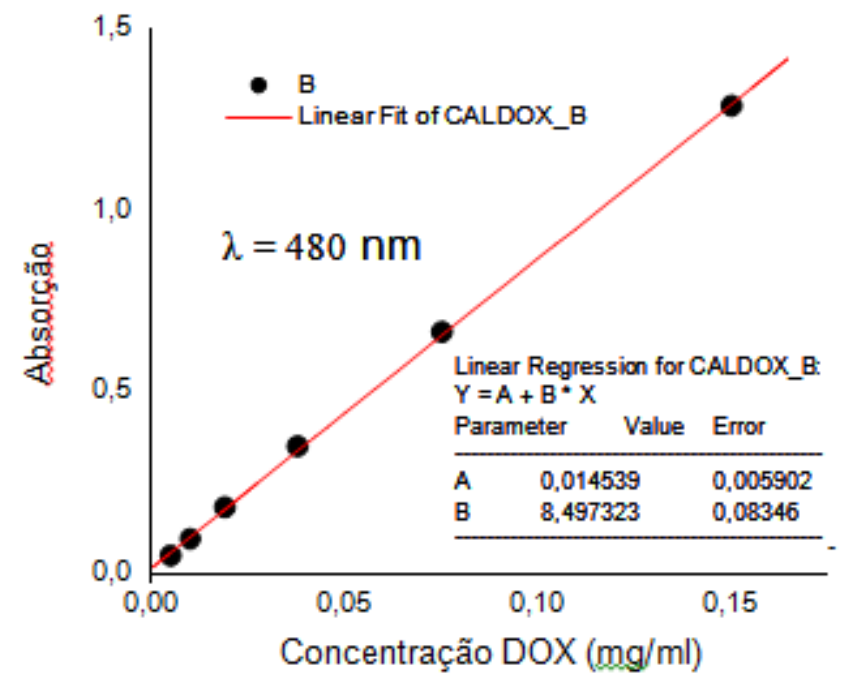

Figura 1: Medidas de absorbância de soluções padrões contendo doxorrubicina (DOX)

\section{3- Resultados e discussão}

\section{1- Caracterização da magnetita}

A magnetita sintetizada pelo método 1 apresentou forte resposta magnética. A figura 2 mostra o difratograma de raios-x dessa amostra. Da medida de DRX e a equação de Scherrer foi determinado o diâmetro médio das partículas, esse valor foi de $30 \mathrm{~nm}$. O difratograma com picos estreitos e intensos é típico de partículas com tamanho dessa ordem de grandeza. As partículas apresentaram parâmetro de rede de 0,8393 nm, valor é bem próximo do parâmetro da magnetita pura, a= 0,8395 nm [4]. 


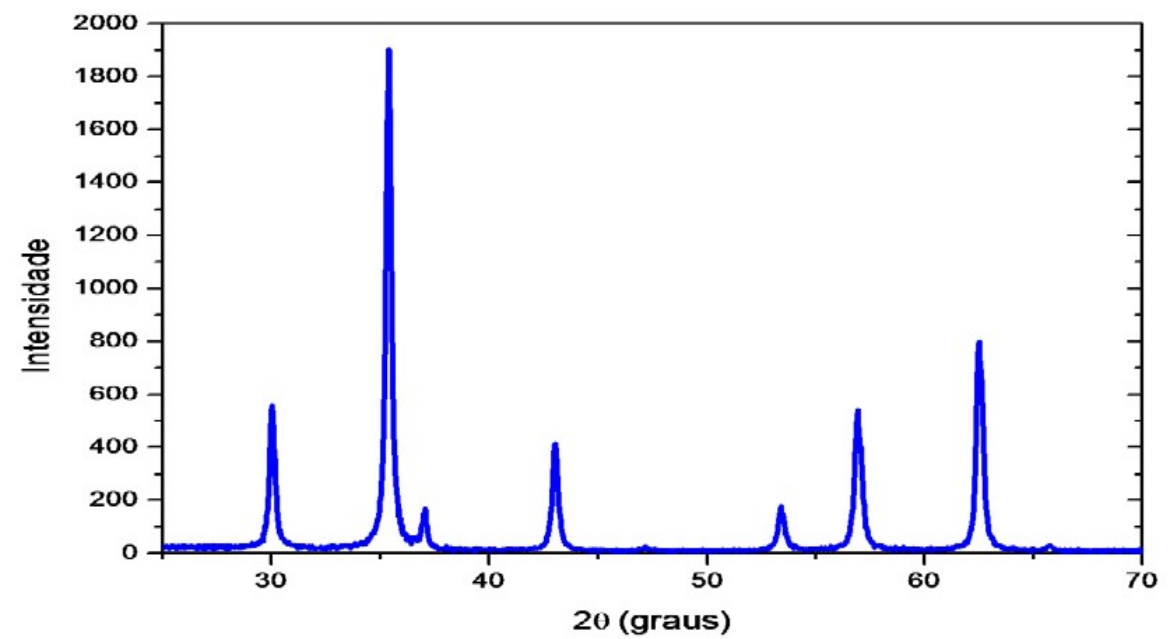

Figura 2: DRX das nanopartículas de magnetita, Amostra1

Outra técnica utilizada para verificar a qualidade da magnetita foi a espectroscopia Mössbauer, figura 3. As medidas de EM foram realizadas a 300K. O espectro Mössbauer mostrou dois sextetos: um deles devido à a interação hiperfina magnética do núcleo de $\mathrm{Fe}^{3+}$ nos sítios $\mathrm{A}$ (sexteto da cor azul), e outro devido a interação hiperfina magnética do núcleo de $\left[\mathrm{Fe}^{3+}+\mathrm{Fe}^{2+}\right]$, nos sítios $\mathrm{B}$ (sexteto da cor verde), da estrutura do espinélio invertido $\left(\mathrm{AB}_{2} \mathrm{O}_{4}\right)$. Nessa estrutura a quantidade de íons de Fe localizados no sítio B é o dobro do sítio A. Dos ajustes, as áreas relativas dos sextetos da cor verde e cor azul foram de 66\% e 34\%, esses valores são proporcionais à quantidade de íons de Fe localizados nesses sítios. Esse resultado mostrou que a qualidade da magnetita produzida é muito boa [4].

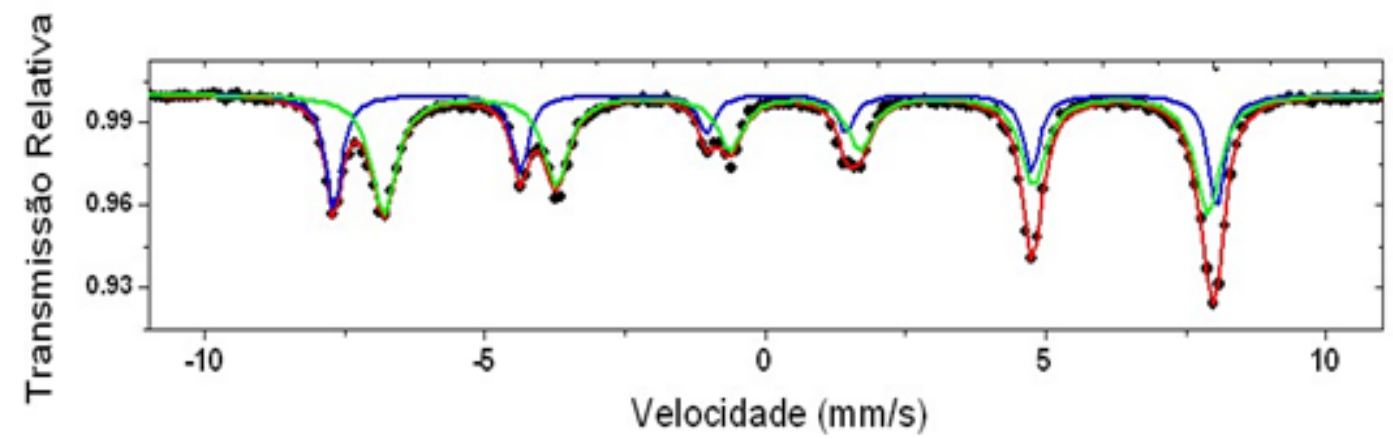

Figura 3: Espectro Mössbauer da magnetita preparada pelo método 1.

A magnetita que foi sintetizada pelo método 2, a medida de DRX mostrou picos largos indicando que as partículas têm diâmetro menor que as partículas do metodo 1 , o valor do diâmetro médio obtido dos ajustes foi de $7 \mathrm{~nm}$, o parâmetro de rede foi de a= 0.8365 $\mathrm{nm}$, sendo esse valor próximo ao parâmetro da magemita [4]. Ver figura 4. 


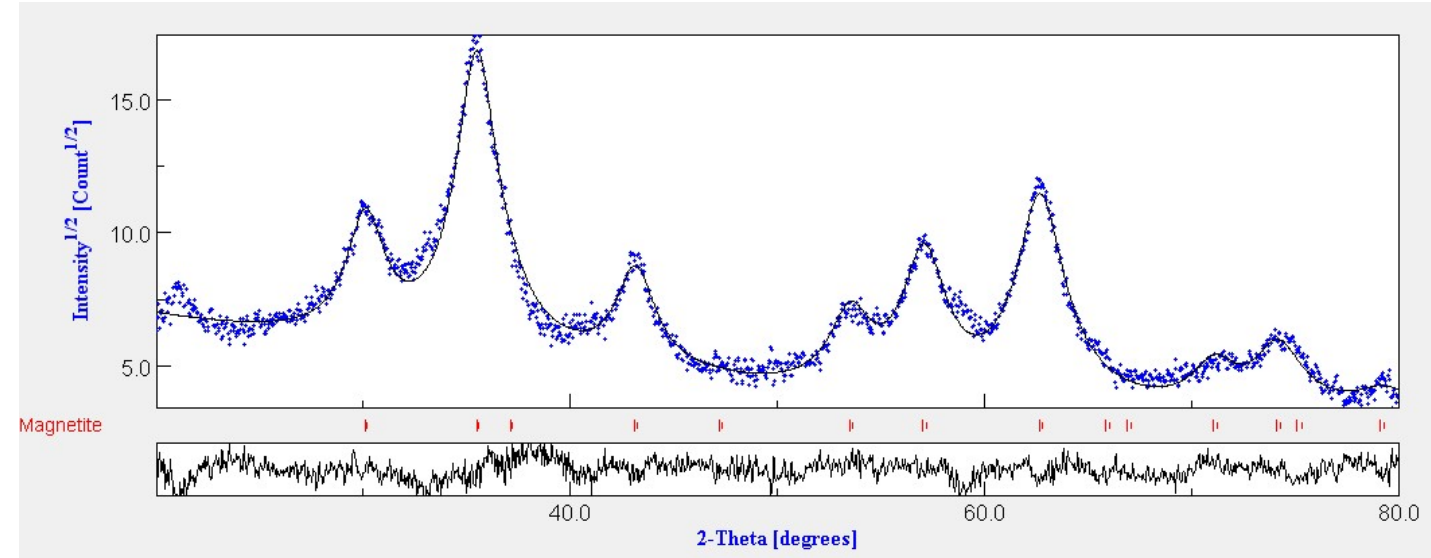

Figura 4: DRX das nanopartículas de magnetita, Amostra2

A medida de EM para esta amostra acusou um espectro superparamagnético tipo dobleto, e não será mostrado.

\section{2- Caracterização das partículas de magnetita revestida com quitosana}

As nanopartículas de quitosana contendo magnetita preparada pelo método 1 (Amostra1) foram caracterizadas pela técnica de EDL. A figura 5 mostra a distribuição de tamanhos dessas partículas. O tamanho médio das partículas variou entre 400-500nm. O elevado valor do diâmetro dessas partículas é devido à agregação das nanopartículas de magnetita, isto é um efeito do elevado valor de momento magnético e as forças atrativas entre os dipolos magnéticos.

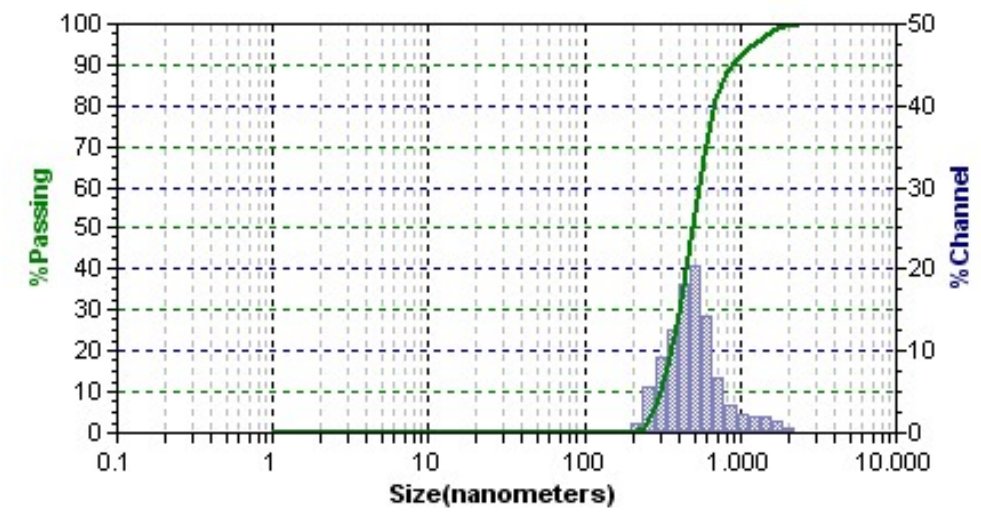

Figura 5: Medidas de EDL para as nanopartículas da Amostra1

As nanopartículas de quitosana contendo magnetita preparada pelo método 2 (Amostra2) também foram caracterizadas pela técnica de EDL. A figura 6 mostra a distribuição de tamanhos. O tamanho médio das partículas variou entre 200-300nm, esse tamanho está de acordo com os objetivos deste trabalho [5]. Já as particulas da amostra Amostra1 têm diâmetro maior e não serão utilizadas para a encapsulação da doxorrubicina. 


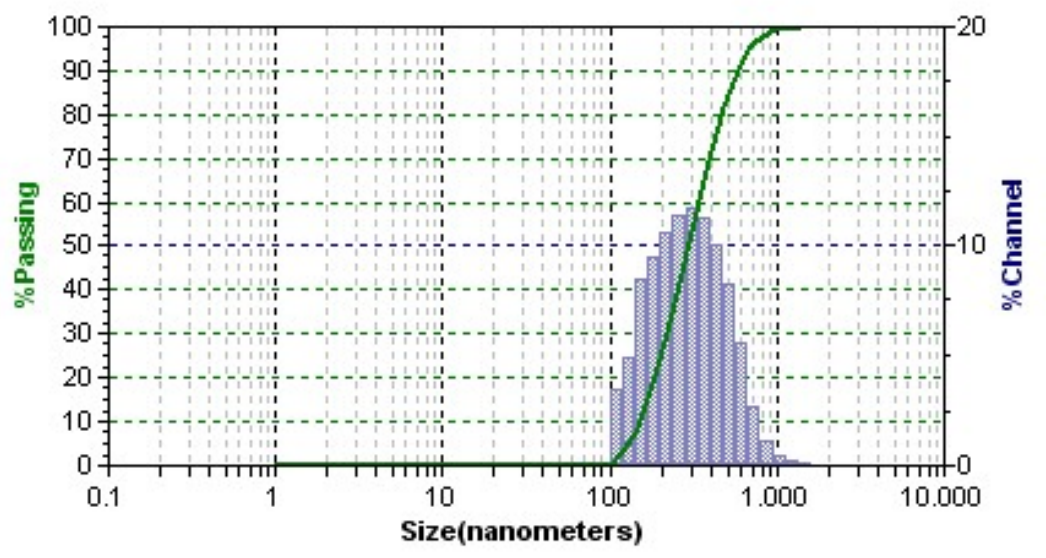

Figura 6: Medidas de EDL para as nanopartículas da Amostra2

\subsection{Estudo da cinética de liberação do fármaco}

Duas amostras foram preparadas utilizando os parâmetros da tabela 1, as medidas de espectrofotometria foram realizadas em diferentes intervalos de tempo. As figuras para as duas amostras preparadas são apresentadas na Figura 7. A suspensão aquosa das partículas foi mantida em um ambiente escuro para evitar a fotodegradação da doxorrubicina, e foi exposta à luz apenas quando foram feitas as medidas. Para fazer as medidas as suspensões foram centrifugadas em 10k RPM por 1 minuto, e $1 \mathrm{ml}$ do sobrenadante foi utilizado para a análise, a seguir esse volume de líquido foi retornado ao recipiente original e submetido a agitação no vortexer por 30 segundos, a suspensão foi mantida em repouso até a próxima medida. Obsevouse que depois de 20 horas a absorção das amostras tende a diminuir, esse fato pode ser devido a adsorção do fármaco nas paredes do recipientes utilizados e a sua fotodegração. Na literatura foi reportado que a doxorrubicina mostra sinais de fotodegradação depois de 24 horas e exposta à luz [6]. Os resultados desses experimentos são apresentados na tabela 2 .
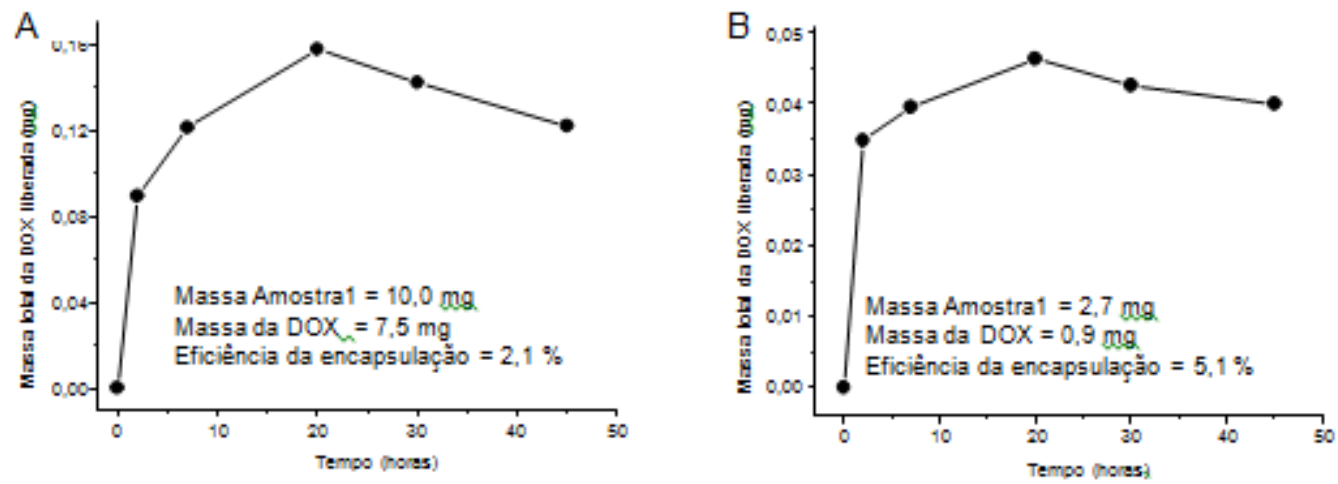

Figura 7: Medidas de espectrofotômetria das amostras (A) AmostraDox1 e (B) AmostraDox2 


\begin{tabular}{|c|c|c|c|}
\hline & A (\%) & B (\%) & C (\%) \\
\hline AmostraDox1 & 75 & 1.6 & 2,1 \\
\hline AmostraDox2 & 33 & 1,7 & 5,1 \\
\hline
\end{tabular}

Tabela 2- Parâmetros relacionados à encapsulação da doxorrubicina.

A) (Massa de Dox utilizada/massa de Amostra1)x 100\%

B) (Massa de Dox Encapsulada*/massa de Amostra1)x

100\% C) Eficiência da encapsulação = A/B x 100\%

*) Massa de Dox Encapsulada = Massa total da Dox liberada**

**) Valor obtido das medidas de espectrofotômetria.

No entanto a relação da massa de Dox encapsulada por grama de amostra1 para as amostras AmostraDox1 e AmostraDox2 são aproximadamente iguais (coluna B), a eficiência de encapsulação da Dox (coluna C) foi maior quando a razão entre as massas da Dox utilizada e a amostra1 foi menor. Esse resultado indica que o parâmetro $C$ pode ser maior se for utilizado uma quantidade menor da Dox. O parâmetro B parece indicar um valor limite para a massa da Dox que pode se encapsulada pelas partículas da amostra Amostra1. Os resultados mostram que ainda não foi atigido o máximo da eficiência da encapsulação da Dox, novos testes devem ser feitos para estudar esse parâmetro.

\section{Conclusão}

Com o método 2 de síntese da magnetita foram produzidas nanopartículas com tamanho médio $7 \mathrm{~nm}$ e quando estas foram funcionalizadas com quitosana e o polímero Pluronic F127 o tamanho médio foi de $250 \mathrm{~nm}$.

Foi encapsulada a Dox e fizemos testes para medir a quantidade de Dox encapsulada, e a eficiência da encapsulação. Duas amostras foram estudadas, os resultados indicaram que há um limite para a quantidade máxima de Dox que pode ser encapsulada. A eficiência da encapsulação pode ainda ser melhorada, outros testes devem ser feitos para determinar o máximo desse parâmetro. O sistema composto de nanopartículas de quitosana magnética e a doxorrubicina é promisor em aplicações que envolvem o tratamento de tumores neoplásicos via a vectorização magnetica. 


\section{Composite Magnetic Polymeric Nanoparticles for use as a Delivery System of Antineoplasic Drugs}

Abstract: Due to the harmful effects of antineoplastic drugs on healthy tissues, new methods are being developed for the vectorization of these drugs. The magnetic vectoring consists in directing magnetic composite nanoparticles through the blood flow to the tumor region, using an external magnetic field gradient. The composite is formed by magnetite nanoparticles and neoplastic drug which are inserted in the matrix of chitosan. Thus, the objective of this work is to synthesize Chitosan particles functionalized with the polymer Pluronic F127 and magnetite nanoparticles as a delivery system of antineoplasic drugs. Two methods were used to prepare the magnetite nanoparticles. The first is the oxidation and precipitation of $\mathrm{Fe}^{2+}$ ions in an alkaline medium of $\mathrm{KOH}$ and $\mathrm{KNO}$, and nitrogen gas atmosphere, the second method consists of stoichiometric mixture of $\mathrm{Fe}^{2+}$ and $\mathrm{Fe}^{3+}$ ions in an alkaline medium $\mathrm{NaOH}$. The magnetite nanoparticles were encapsulated in chitosan matrix using a method developed in laboratory. The samples were characterized using the techniques of $x$-ray diffraction (XRD), Mössbauer spectroscopy (MS) of 57Fe, dynamic light scattering (DS) and spectrophotometry UWis. The XRD measurements performed at 300K and MS showed formation of magnetite nanoparticles with diameters between 7 and $30 \mathrm{~nm}$. The EDL measurements showed size distributions of magnetite particles coated with chitosan with an average diameter of 250 and $450 \mathrm{~nm}$. This system has potential for use in cancer therapy.

Keywords: Câncer, Magnetita, Quitosana.

\section{Clique aqui para digitar texto.}

\section{5- Referências}

1- NASCIMENTO A., LARANJEIRA M.C., FAVERE, V. T., JOSUE A. J Microencapsul.,v.3, n.5 p.679-684,2001.

2- OKON, E., POULIQUEN, D., OKON, P.; KOVALEVA, Z. V., STEPANOVA, T. P.;

LAVIT, S. G., Kudryavtsev, B. N., Jallet, P. Lab. Invest. 71, 895-90, 1994.

3- YUAN, F., DELliAN, M., FUKUMURA, L. M., BERK D. A., TORCHILIN, V. P., Jain, R. K. Cancer Res. 55, 3752, 1995.

4- SCHEWERTMANN U., CORNELL R.M. Iron Oxides in the Laboratory: Preparation and Characterization, Wiley, 2000.

5- MAEDA H., WU J., SAWA T., MATSUMURA Y., HORI K., J. Control. Release 65, 271-284, 2000

6- CARMICHAEL A.J., RIESZ P., Arch. Biochem. Biophys., v. 237, 2, 433-

444 , 
